\title{
Distinct roles for strigolactones in cyst nematode parasitism of Arabidopsis roots
}

\author{
Carmen M. Escudero Martinez • Nina Guarneri • Hein Overmars • Casper van Schaik • \\ Harro Bouwmeester • Carolien Ruyter-Spira • Aska Goverse
}

Accepted: 5 February 2019 / Published online: 18 February 2019

(C) The Author(s) 2019

\begin{abstract}
Phytohormones play an essential role in different stages of plant-nematode interactions. Strigolactones (SLs) are a novel class of plant hormones which play an important role in plant development. Furthermore, certain soil-inhabiting organisms exploit this plant molecule as allelochemical. However, whether SLs play a role in plant parasitism by nematodes is as yet unknown. This prompted us to investigate the potential role of SLs in different stages of the nematode life cycle using the beet cyst nematode Heterodera schachtii and Arabidopsis as a model system. We
\end{abstract}

C. M. Escudero Martinez • N. Guarneri · H. Overmars •

C. van Schaik · A. Goverse $(\bowtie)$

Laboratory of Nematology, Plant Science Group, Wageningen

University, Droevendaalsesteeg 1, 6708 PB Wageningen, the

Netherlands

e-mail: aska.goverse@wur.nl

C. M. Escudero Martinez

Cell and Molecular Science, James Hutton Institute, Invergowrie,

Dundee DD2 5DA, UK

H. Bouwmeester · C. Ruyter-Spira

Laboratory of Plant Physiology, Plant Science Group,

Wageningen University, Droevendaalsesteeg 1, 6708

PB Wageningen, the Netherlands

H. Bouwmeester

Swammerdam Institute for Life Sciences, University of

Amsterdam, Science Park 904, 1098 XH Amsterdam, the

Netherlands

C. Ruyter-Spira

Science Groups, Wageningen University, Droevendaalsesteeg 1,

6708 PB Wageningen, the Netherlands analyzed the effect of SLs on cyst nematode hatching, host attraction and invasion, and the establishment of a feeding relation upon infection of the SL deficient mutant $\max 4-1$ and the SL signaling mutant $\max 2-1$. In addition, infection assays were performed under phosphate shortage to enhance SL production and in the presence of the synthetic SL analog GR24. From this study, we can conclude that SLs do not contribute to cyst nematode hatching at the levels tested but that they do play a role in host attraction and subsequent invasion in a MAX2 dependent manner. Furthermore, we observed that increased levels of exogenous and endogenous SLs change the root invasion zone. Upon root infection, cyst nematode development was enhanced in both the $\max 2-$ 1 and $\max 4-1$ mutants due to the formation of enlarged feeding cells. These data provide evidence for distinct roles of SLs during cyst nematode parasitism of plant roots.

Keywords Strigolactones $\cdot$ Allelochemical $\cdot$ Heterodera schachtii $\cdot$ Syncytium $\cdot$ Nematode feeding site .

Arabidopsis

\section{Introduction}

Cyst nematodes (CN) of the genera Heterodera and Globodera belong to the most harmful plant-parasitic nematodes for a number of major food crops (Jones et al. 2013). Worldwide annual crop losses caused by plant-parasitic nematodes have been estimated to exceed $\$ 157$ billion U.S. dollars (Abad et al. 2008). In high 
densities, these nematodes disrupt the physiology of their host plant, decreasing their fitness and therefore causing reductions in crop yield and quality. General symptoms associated with these nematodes are stunted plants, arrested growth and nutrient deficiencies (Akintayo et al. 2018). Additionally, specific symptoms are the cutinized cysts found either along the root surface or in the soil. They remain in the soil for decades and make the control of cyst nematodes difficult and complex (Jones et al. 2013).

To improve existing control measures or to develop novel strategies it is necessary to have a better understanding of the mechanisms involved in the cyst nematode's life cycle, since every step is a potential target to disrupt nematode parasitism on plants. The first step is the hatching of infective juveniles from the eggs, which are protected by the cyst against adverse environmental conditions (Turner and Rowe 2006). This process depends on or is enhanced by hatching factors released by plant roots, which allows the cyst nematode to synchronize with their host (Riga et al. 2001; Turner and Rowe 2006; Ito et al. 2015). Upon hatching, the pre-parasitic second stage juveniles (J2) are attracted to the host roots by unknown factors (Grundler et al. 1991). Once they have reached the roots, they enter the root preferably in the elongation zone (von Mende 1997) and migrate intracellularly by mechanical stylet thrusting and enzymatic break down of plant cell walls by secretions from the subventral glands (Tytgat et al. 2002). Subsequently, they induce a specialized feeding cell known as syncytium on which they fully depend for their development and reproduction. Once these amphimictic nematodes have moulted into $\mathrm{J} 3$ and $\mathrm{J} 4$, the vermiform adult males leave the roots to mate with the females. Upon fertilization, the $\mathrm{J} 1 / \mathrm{J} 2$ develop inside the eggs which are contained by the swollen female body. When the female dies, the cuticle of the dead body forms a resistant darkbrown cyst that protects the eggs in the soil (Grundler and Wyss 1992).

Cyst nematodes are obligatory sedentary endoparasites that establish a permanent feeding site in the vascular bundle of the host roots that functions as a highly active metabolic sink to provide nutrients to the developing nematode. In the initiation and development of the syncytium plant hormones play an essential role (Cabrera et al. 2015). The first feature of the initial syncytial cell is a progressive enlargement of the nucleus due to DNA endoreduplication (de Almeida Engler et al. 2015). Subsequently, callose depositions can be seen in the cell wall of this and neighbouring cells (Hofmann et al. 2010). Other unique characteristics of a syncytium are the observed decrease in volume of the central vacuole which coincides with an increase in organelle number and cytoplasmic density. Furthermore, partial cell wall dissolution allows the fusion of neighbouring cells and feeding cell expansion. Typically, cell wall ingrowths are formed along the xylem to increase the surface for nutrient uptake (Holtmann et al. 2000; Rodiuc et al. 2014). The mature syncytium comprises about 200 cells via incorporation of surroundings procambial cells (Sobczak and Golinowski 2011). Several studies have shown that phytohormones like auxin and ethylene play a crucial role in the formation of syncytia and that cyst nematodes are able to manipulate the hormone balance in host roots as well as their response pathways (Goverse and Bird 2011; Cabrera et al. 2015).

Strigolactones (SLs) are carotenoid-derived terpenoid lactones and are a relatively new group of plant hormones (Umehara et al. 2008; Gomez-Roldan et al. 2008). Orthologous genes implicated in the SL biosynthesis pathway have been identified in monocot and dicot plant species such as rice, petunia, tomato, pea and Arabidopsis (Al-Babili and Bouwmeester 2015). SLs are involved in the inhibition of shoot branching (Gomez-Roldan et al. 2008), secondary stem growth and leaf senescence (Al-Babili and Bouwmeester 2015) and seem to play a role in reproductive development (Kohlen et al. 2011b). In addition, SLs are important regulators of root development with effects on the formation of primary roots (Ruyter-Spira et al. 2011), lateral roots (Kapulnik et al. 2011; Ruyter-Spira et al. 2011) and root hairs (Kapulnik et al. 2011). Furthermore, SLs are involved in the chemical communication of plants with several groups of organisms in the rhizosphere to establish either symbiotic or parasitic relationships with their host. For example, SLs are germination stimulants for parasitic plants of the genera Orobanche and Striga spp. (Cook et al. 1972). They also act as hostrecognition signals and branching stimulants for arbuscular mycorrhizal fungi (AMF) (Akiyama et al. 2005). Moreover, SLs have been related to interactions between Rhizobium and legumes, since nodulated plants trigger less germination of Orobanche spp. parasite weeds, suggesting reduced levels of SLs in those nodulated plants (Mabrouk et al. 2007).

Despite their role in root development and importance for several soil organisms, the role of SLs in the 
relation between plant-parasitic nematodes and their hosts is still unknown. A phytopathological study should be based on knowledge of both the physiology of the host plant and its parasite (after Prof. dr. Johanna Westerdijk 1917). With this in mind, we decided to investigate the possible role of SLs in different stages of cyst nematode parasitism like hatching, host attraction, host invasion and feeding site development, as well as nematode maturation. For this, the interaction between the beet cyst nematode Heterodera schachtii and the plant species Arabidopsis was used as model. In Arabidopsis, several mutants are available for genes involved in SL biosynthesis and signal transduction pathways. For this study, the SL deficient mutant max4-1 (Sorefan et al. 2003) was selected to test the role of SL biosynthesis in cyst nematode parasitism. The SL signal transduction mutant $\max 2-1$ (Stirnberg et al. 2002) was selected to investigate the role of SL mediated signalling in cyst nematode parasitism in the Col-0 background. Moreover, the synthetic SL analogue GR24 was used to test the role of SLs in cyst nematode hatching, as well as a control for SL deficient and insensitive Arabidopsis mutants in the nematode infection assays.

\section{Materials and methods}

\section{Hatching experiment}

Cysts from Heterodera schachtii were collected from 4 months to 6 months old cabbage plants (Brassica oleracea var. gemmifera cv. Cyrus) from a nematode culture in the greenhouse and exposed to different treatments of the synthetic SL GR24, using decreasing concentrations to construct a dose-response curve to test the effect on juvenile hatching. These treatments consisted of a series of log dilutions of GR24 from $10^{-6}$ to $10^{-10}$ $(0,3 \mu \mathrm{M}, 0,03 \mu \mathrm{M}, 0,003 \mu \mathrm{M}, 0,0003 \mu \mathrm{M})$ based on the concentrations that stimulate germination in parasitic plants $\left(10^{-7}-10^{-15} \mathrm{M}\right)$ (Wigchert and Zwanenburg 1999). The cysts were collected by Cobb's method and a batch of ten cysts was selected per treatment, discarding immature females or partially empty cyst, and transferred to different hatching chambers (Steele 1972). The controls for this experiment were demineralised water, $0,3[\mathrm{v} / \mathrm{v}]$ acetone in demineralised water (as GR24 is diluted in acetone) and root exudates from six weeks old kale plants (Brassica oleracea var. sabellica), which is a host for $H$. schachtii. Three replicates were performed for each treatment and control, and the number of hatched J2 was counted weekly under stereo microscope (Leica Microssytems) for a total of 8 weeks. At the end of the experiment the cysts were crushed in order to count the remaining eggs and to determine the percentage of hatching for each batch. The experiment was carried out twice at a temperature of $20 \pm 2{ }^{\circ} \mathrm{C}$.

\section{Arabidopsis plant material}

Seed from the Arabidopsis mutant, max2-1 (Stirnberg et al. 2002), $\max 4-1$ (Sorefan et al. 2003), Columbia-0 wild-type lines were vapour sterilized and the seeds were placed on agar plates containing Murashige and Skoog culture medium without sucrose $(0,5 \mathrm{MS}, \mathrm{pH} 6,4)$ for germination. Seedlings were grown at $24{ }^{\circ} \mathrm{C}$ with $16 \mathrm{~h}$ of light and transferred to different media for nematode infection studies.

Infective nematode juveniles

Cyst nematodes of the species $H$. schachtii were collected from 4 months to 6 months old cabbage plants (Brassica oleracea var. gemmifera cv. Cyrus) from the nematode culture in the greenhouse. Cysts were extracted by Cobb's sieving method and placed in a hatching solution of $\mathrm{ZnCl}_{2}(3 \mathrm{mM})$ for 3-7 days in the dark to obtain pre-parasitic J2. Upon hatching, they were sucrose purified and surface sterilized by incubating the pre-parasitic juveniles for $20 \mathrm{~min}$ in streptomycinpenicillin $(0,5 \% \mathrm{w} / \mathrm{v})$ and $20 \mathrm{~min}$ in ampicilingentamicyn $(0,1 \% \mathrm{w} / \mathrm{v})$. Finally, they were immerged in a chlorhexidine $(0,1 \% \mathrm{v} / \mathrm{v})$ solution for $3 \mathrm{~min}$ followed by extensive washing in sterile tap water (after Postma et al. 2012). Antibiotics and chlorhexidine were obtained from Duchefa Biochemie B.V. and sucrose was purchased from the grocery store.

In vitro nematode infection assays

One-week old seedlings (24 per genotype Col-0, $\max 4$ 1 or $\max 2-1)$ were transferred to 12 well-plates filled whit KNOP medium (Sijmons et al. 1991) without sucrose and maintained at $24{ }^{\circ} \mathrm{C}$ with $12 \mathrm{~h}$ of light. In addition, the same conditions were used to perform infection assays in medium without phosphate (KNO(P)) to enhance SL production (López-Ráez et al. 2008; 
Kohlen et al. 2011a) and KNOP medium supplied with the synthetic strigolactone GR24 in a concentration of $1 \mu \mathrm{M}$ (after Ruyter-Spira et al. 2011). After one week, approximately 150 surface sterilized pre-parasitic J2 were inoculated per plant at the agar surface next to the root tips in each well. The infected plants were monitored 1, 7, 14 and 28 days post inoculation (dpi) under a stereo microscope (Leica Microsystems, Wetzlar, Germany) to observe host attraction, host penetration and feeding site development. To obtain quantitative data nematodes were counted by visual scoring of the roots using the stereo microscope.

\section{Statistical analysis}

The statistical analyses for the comparison of one variable between two groups was performed by a t-test with equal variance in Microsoft Excel (2007). Conversely, for the comparison of more than two groups for one variable the analysis was done in SPSS; this was attraction, penetration and susceptibility. Normality of the data was checked by applying the KolmogorovSmirnov non parametric test $(p=0.05)$. Homoscedasticity of the data was checked by applying the Levene test $(p=0.05)$. The assumptions of normality and homoscedasticity were fulfilled before accomplishing one way ANOVA. This test compares the variance of the control and treatments, the post-hoc test was Duncan's multiple mean comparison test and multiple comparisons by Games-Howell.

\section{Results}

No effect of the SL analogue GR24 on the hatching of $H$. schachtii from cysts

Several soil-inhabiting organisms like parasitic plants and mycorrhizal fungi use strigolactones (SLs) produced by roots of their host plant as germination stimulants (Cook et al. 1966; Akiyama et al. 2005). Therefore, we tested if SLs could also potentially act as hatching factor for $H$. schachtii by evaluating the hatching activity of the synthetic SL analogue GR24. However, exposure of cysts to different dilutions of GR24 resulted in hatching percentages that were not significantly different from the negative water controls (Fig. 1). Stimulation of nematode hatching was only significantly induced upon application of kale root diffusate as a positive control (Fig. 1).

Host attraction and penetration of infective juveniles involves MAX2-dependent SL signalling

To test whether SLs play a role in the next stage of cyst nematode parasitism, the effect on host attraction and root invasion was investigated. Therefore, an infection assay was performed on the Arabidopsis SL biosynthesis mutant max4-1 and the SL signal transduction mutant max2-1 using wild type Arabidopsis Col-0 as a control. The $\max 2-1$ and $\max 4-1$ mutants both showed increased number of lateral roots compared to the wild type plants, consistently with what was previously described (Ruyter-Spira et al. 2011; Kapulnik et al. 2011). At 1 day post inoculation (dpi), no difference in host attraction was observed for the max4-1 mutant compared to the wild type. However, a significant decrease in number of preparasitic J2 was observed near the roots of the max2-1 plants compared with the wild type control and max4-1 mutant (Table 1). This suggests that host attraction of infective juveniles of the cyst nematode H. schachtii involves - MAX2 mediated - SL signalling, but not - MAX4 dependent - SL biosynthesis. This was further supported by the observation that no significant differences were observed in infective juvenile attraction to the roots when wild type plants were grown on medium complemented with synthetic SL (+GR24) or under phosphate shortage (P) to enhance the endogenous SL production and exudation (Table 1) (López-Ráez et al. 2008). Also for the max4-1 mutant, no significant change in host attraction was observed under these conditions. For the $\max 2-1$ mutant, however, attraction to the roots was slightly enhanced under $-\mathrm{P}$ although not statistically significant.

Next, we investigated whether SLs play a role in host invasion of the roots by the infective juveniles. No significant change in root penetration was observed for the max4-1 biosynthesis mutant compared to the wild type control at $1 \mathrm{dpi}$ when grown on standard KNOP medium, nor under phosphate shortage and GR24 complementation (Table 1). This shows that host invasion by cyst nematodes does not depend on MAX4 mediated SL biosynthesis. However, for the $\max 2-1$ signalling mutant a significantly smaller number of nematodes was 


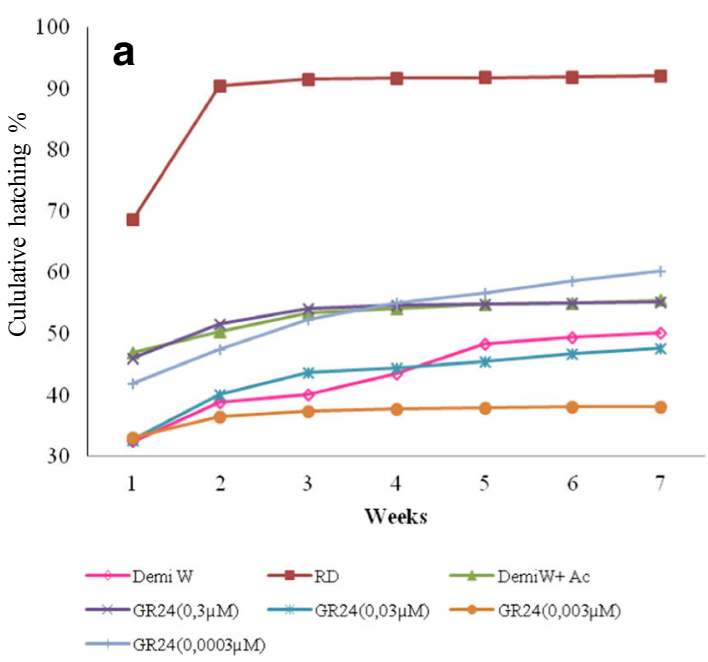

Fig. 1 Effect of the synthetic SLs analogue GR24 on nematode hatching: a Cumulative percentage of pre-parasitic J2 from $H$. schachtii which were hatched in a series of logarithmic GR24 dilutions (from $0,3-0,0003 \mu \mathrm{M}$ ) during 7 weeks of exposure. The controls were kale root diffusates (RD, positive control), demineralised water (Demi $\mathrm{W}$ ) and demineralised water with

detected inside the roots at $1 \mathrm{dpi}$ (Table 1 ). This seems to correlate with the observed reduction in attraction by this mutant at $1 \mathrm{dpi}$, which most likely explains the observed reduction in root penetration of the $\max 2-1$ plants. So, these data indicate that both host attraction and subsequent host invasion by cyst nematode juveniles involves MAX2 mediated signalling. Interestingly, host invasion of the $\max 2-1$ mutant by infective juveniles was restored to wild type levels

Table 1 Host attraction and host invasion of $H$. schachtii $\mathbf{J} 2$ on the SLs insensitive mutant $\operatorname{max2}-1$ and SLs biosynthesis mutant max4-1 compared to Col-0 wild type plants on standard KNOP medium or under phosphate shortage (-P) or GR24 complementation: (A) Total nematode numbers were counted and the mean values of nematode attraction (left) and penetration (right) one day post inoculation (dpi) are shown

\begin{tabular}{llllllll}
\hline 1 dpi & \multicolumn{2}{l}{ Attraction } & & \multicolumn{2}{l}{ Penetration } \\
\cline { 2 - 4 } \cline { 6 - 7 } \cline { 5 - 6 } & Col-0 & $\max 4$ & $\max 2$ & & Col-0 & $\max 4$ & $\max 2$ \\
\hline KNOP & $52,18^{\mathrm{a}}$ & $53,89^{\mathrm{a}}$ & $35,83^{\mathrm{a} *}$ & $17,94^{\mathrm{a}}$ & $23,67^{\mathrm{a}}$ & $11,17^{\mathrm{a} *}$ \\
-P & $44,22^{\mathrm{a}}$ & $52,35^{\mathrm{a}}$ & $49,17^{\mathrm{a}}$ & $23,44^{\mathrm{a}}$ & $23,94^{\mathrm{a}}$ & $24,17^{\mathrm{b}}$ \\
GR24 & $46,73^{\mathrm{a}}$ & $43,50^{\mathrm{a}}$ & $40,19^{\mathrm{a}}$ & $14,40^{\mathrm{a}}$ & $17,13^{\mathrm{a}}$ & $12,13^{\mathrm{a}}$ \\
\hline
\end{tabular}

Letters symbolize significant differences of the same plant genotype performing under different media $(\mathrm{p}<0,05)$. Asterisks represent significant differences between the WT, $\max 4-1$ and $\max 2-1$ $(p<0,05)$

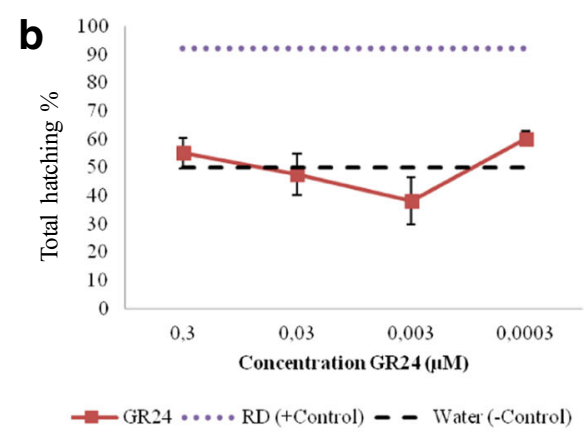

acetone (Demi W + Ac, negative controls). Error bars are not shown for visualization reasons. b Emergence of pre-parasitic J2 in tenfold dilutions of GR24 (from $0,3-0,0003 \mu \mathrm{M}$ ). The horizontal dotted line indicates the percentage of hatching in root diffusates and the horizontal dashed line indicates the maximum percentage of hatching in water

when grown under phosphate starvation (-P), which suggests a role for endogenous SLs in root invasion independent of MAX2. This effect was not observed when the synthetic SL analogue GR24 was exogenously applied to the max2-1 plants, consistent with the SL insensitive phenotype of this mutant.

Increased SL levels change the root invasion zone in a MAX2 dependent manner

Inoculation of wild type Arabidopsis plants with a high density of pre-parasitic juveniles revealed that after $1 \mathrm{~h}$ the nematodes were attracted towards a specific area of the root close to the tip of the primary and lateral roots (Fig. 2). For the $\max 2-1$ and $\max 4-1$ mutant, a similar aggregation around the root tip was observed. Interestingly, when wild type Arabidopsis plants were grown under phosphate starvation or in the presence of the synthetic SL analogue GR24 the nematodes did not accumulate in these defined regions, but seem to spread along the roots instead. This phenomenon was also observed in the $\max 4-1$ mutant, but not for the $\max 2-1$ mutant in the presence of GR24 or phosphate starvation consistent with the SL insensitive phenotype of this mutant. Host attraction to these regions was followed by subsequent penetration of the roots, from which we conclude that an increase in both exogenous 


\section{Col-0}
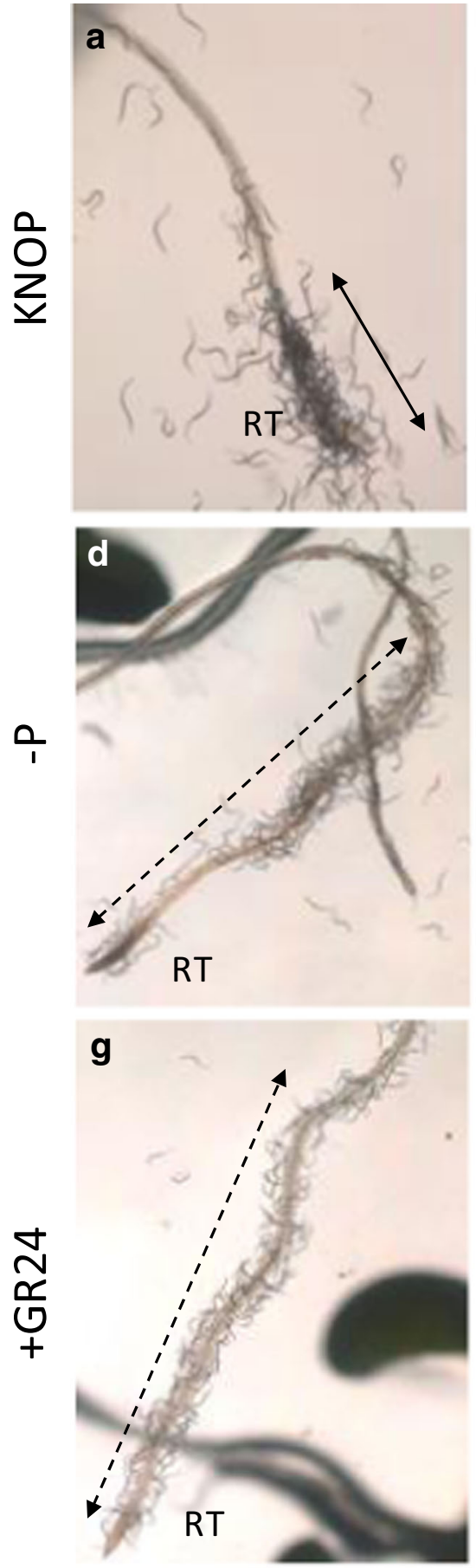

Fig. 2 Effect of phosphate shortage (-P) and GR24 (1 $\mu \mathrm{M})$ complementation on the nematode root invasion zone of the max2-1 and max4-1 Arabidopsis mutants compared to Col-0 wild type roots when grown in vitro on KNOP medium in 12well plates. For this, a high density of infective juveniles from the $\max 4$
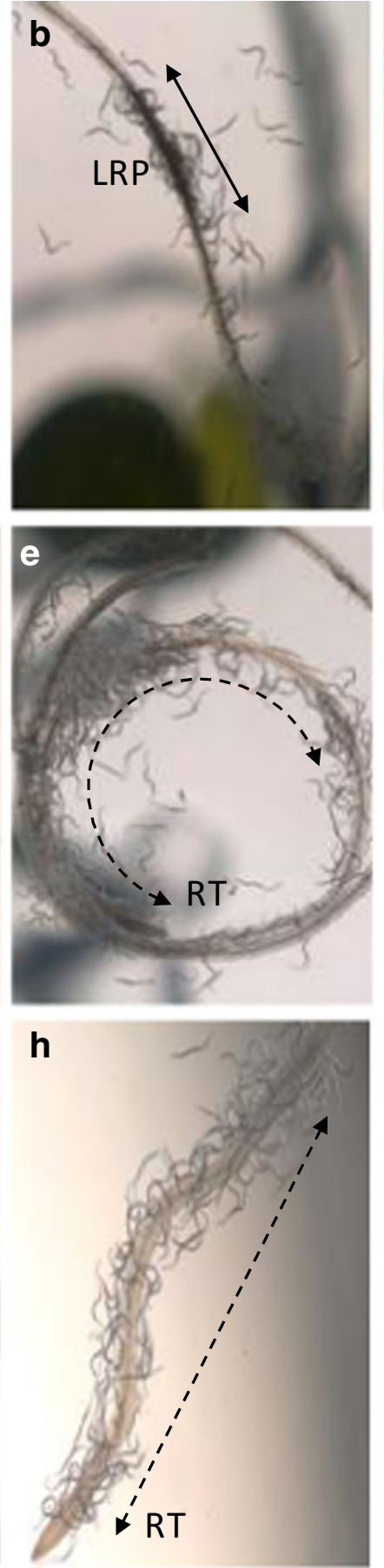

$\max 2$
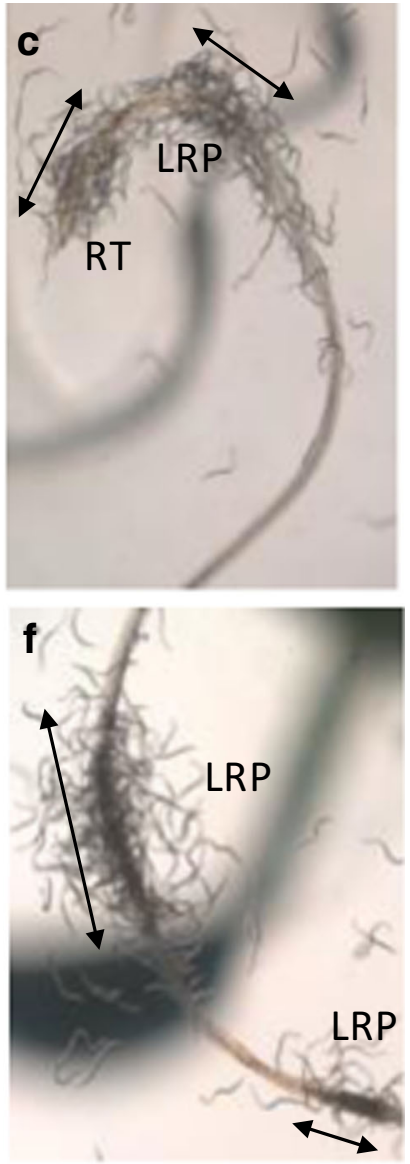

i

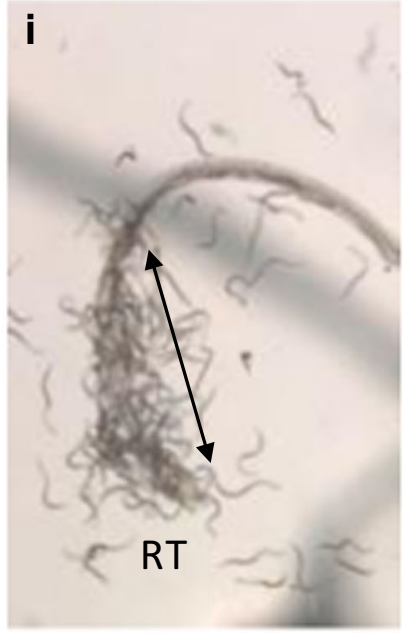

beet cyst nematode $H$. schachtii was applied on the agar surface and host attraction to the roots was monitored $1 \mathrm{~h}$ post inoculation. Aggregation of nematodes along the root invasion zone is marked for each treatment. In total, 24 plants per treatment were observed and representative pictures are shown 
and endogenous SL levels result in the expansion of the root invasion zone in a MAX2 signalling dependent manner.

Syncytium and female development is enhanced in a MAX2 and MAX4 dependent manner

Next, we tested whether SLs play a role in the establishment of a successful feeding relationship by cyst nematodes upon host invasion. Therefore, the total number of adult females was counted at $28 \mathrm{dpi}$ for all combinations and used for statistical analysis. Only for the max21 mutant a significant reduction in the number of females was observed compared to the max4-1 and wild type plants on standard KNOP medium (Fig. 3a). Again, this is most likely due to the observed reduction in host attraction and subsequent invasion. In that case, the data suggest that there are no major obstacles in the successful initiation of a permanent feeding site and their further development once the infective juveniles have invaded the roots of either wild type or mutant plants. However, we could observe enlarged females with a gelatinous egg matrix attached to the posterior part of their body on both the max2-1 and max4-1 mutant compared to the wild type control plants. Moreover, these females were attached to larger and more pronounced syncytia compared to the control plants (Fig. 3b). These data suggest that in both the $\max 2-1$ and $\max 4-1$ mutants the development of feeding sites is promoted resulting in the enhanced development of adult females most likely due to a better food supply. This suggests that cyst nematode feeding cell development involves SLs biosynthesis and signalling in a MAX4 and MAX2 dependent manner, respectively.

\section{Discussion}

Plant allelochemicals are important components for hatching stimulation of cyst nematodes as they enhance
Fig. 3 Nematode and feeding cell development on the max2-1 and max4-1 mutant as compared to Col-0 wild type: a Average number of females developing per plant genotype at 28 dpi on KNOP medium. b Representative image of a normal developed female on Col-0 plants. $\mathbf{c}$ Representative image of a female developed on $\max 4-1$ plants and $\mathbf{d} \max 2$ - plants. Error bars represent standard error. Scale bars $=100 \mu \mathrm{m}$
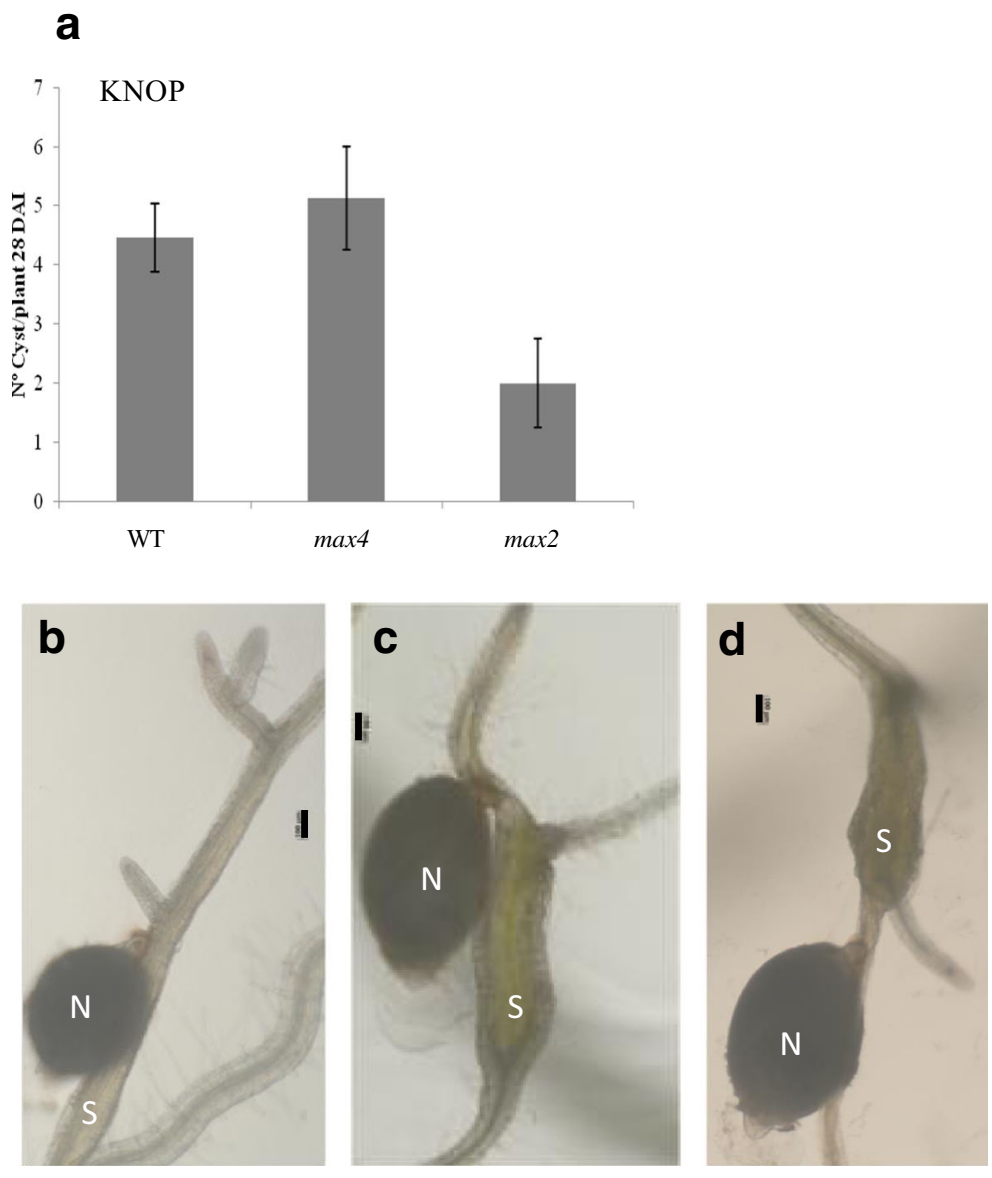
the hatching of most of these endoparasites including H. schachtii (Turner and Rowe 2006). The beet cyst nematode $H$. schachtii has a wide host range, which comprises species of the plant families Chenopodiaceae and Brassicaceae. Hatching of pre-parasitic stage juveniles (J2) from eggs is enhanced by the release of rootdiffusates of these host plants, but not exclusively, as some juveniles spontaneously hatch in water (Clarke and Shepherd 1964). Strigolactones (SL) are functioning as plant allelochemicals for a number of soil-born organisms by for example stimulating germination of parasitic plants (Cook et al. 1972) and hyphal branching of arbuscular mycorrhizal fungi (Akiyama et al. 2005). This prompted us to investigate whether SLs also act as a hatching stimulant for the beet cyst nematode $H$. schachtii. However, the results show that there are no significant differences between hatching in the presence of the synthetic SL analogue GR24 and the negative control, indicating that SLs do not contribute to cyst nematode hatching. The influence of SLs on soil organisms occurs at very low concentrations $\left(10^{-7}-10^{-15} \mathrm{M}\right.$; Wigchert and Zwanenburg 1999). Similarly, nematode hatching factors are also active at very low concentrations in the range of $10^{-14} \mathrm{M}$ (Masamune 1976; Masamune et al. 1982). We used higher GR24 concentrations in the range of $10^{-6}$ to $10^{-9} \mathrm{M}$. GR24 increased swarming of S. melioti (Rhizobium) when applied at 0.1 and $1 \mu \mathrm{M}$ (Peláez-Vico et al. 2016) and at $10^{-8}$ the increased germination of Phelipanche ramosa (Kohlen et al. 2011a) indicating the potential physiological activity of GR24 in the concentration range used in our study.

Upon hatching, the infective cyst nematode juveniles (J2) are attracted towards the plant roots. This process presumably involves host allelochemicals, which are perceived by the chemosensory organs of the nematode (amphids) and allow them to locate and migrate in the soil towards the roots (Curtis 2008). This process is indispensable for the invasion of the host and the establishment of a feeding site within a limited time period after hatching, which is determined by the infective juvenile nematode energy reserves. Therefore, host cues are essential to expedite these processes (Robinson and Perry 2006). Also for parasitic plants, host cues seem to play a role in orientation of radicle growth towards the host root, and the germination stimulants (likely the SLs) have been suggested as a possible candidate for this cue (Dube and Olivier 2001). Interestingly, the phytohormone auxin has been shown to attract nematodes and change their parasitic behaviour (Duncan et al. 1996; Akhkha et al. 2002; Kyndt et al. 2016). Moreover, a putative auxin binding protein was detected in amphidial sections of the potato cyst nematode (Duncan et al. 1996). From these data it was concluded that auxin may act as a cue in the chemical communication between host and parasite (Duncan et al. 1996; Akhkha et al. 2002). Interestingly, there is extensive cross-talk between auxin and SLs in plants roots (review by Koltai 2015). For example, auxin is a positive regulator of SL biosynthesis and SLs on the other hand regulate the plasma-membrane localisation of the auxin-efflux protein PIN2. We observed that the SL signal transduction mutant, $\max 2-1$, displayed significantly less attraction than the biosynthetic mutant. Apparently, host attraction depends on $M A X 2$, which suggests that changes in auxin homeostasis can be detected by the infective juveniles. Therefore, it will be interesting to further investigate the interplay between auxin and SLs as possible host cues in cyst nematode root attraction.

Upon host attraction, cyst nematodes do not randomly invade the roots but they prefer the elongation zone to enter the plant (von Mende 1997). However, the cues involved in the selection and delineation of this specific region by the infective juveniles are virtually unknown. Interestingly, we observed that the exogenous application of the synthetic SL analogue GR24 changed the distribution pattern of the infective juveniles along the root surface upon host attraction. Instead of aggregating at a specific region in the elongation zone the nematodes were now more randomly distributed around the roots in a larger region. A similar effect was observed under phosphate starvation consistent with a role for increased SL levels in shaping the root invasion zone. Moreover, this effect was not observed for the SL insensitive $\max 2-1$ mutant, which shows that the observed modulation of the root invasion zone by increased SL levels depends on MAX2 mediated signalling, either induced by exogenous SLs or endogenous SLs. Increased SL levels apparently deregulate the spatiation of the cyst nematode host invasion zone in a MAX2 dependent manner. This may be the result of changes in the auxin flux in the root upon the modulation of local auxin levels by the application of GR24 or phosphate starvation (Ruyter-Spira et al. 2011). This could suggest that auxin does not only control cyst nematode host attraction through MAX2 mediated signalling but also defines the region where the larvae can enter the plant root. 
Once the infective cyst nematode juveniles locate the root, they will penetrate the root and search for a suitable cell type to settle and induce a permanent feeding structure (Golinowski et al. 1996). The number of fully developed adult female nematodes was reduced on the SL signal transduction mutant, $\max 2-1$ but not on the max4-1 SL biosynthesis mutant. This suggests that the number of parasitic cyst nematodes on Arabidopsis roots is not dependent on MAX4 mediated SL biosynthesis, but may involve MAX2 dependent SL signalling. However, it is more likely that this reduction in the number of parasitic nematodes on the max2-1 mutant is the result of reduced host attraction as observed in this study instead of an arrest in penetration or the establishment of a feeding site. This is further supported by the observation that the nematodes which were able to develop on the max2-1 mutant showed normal progression through the different parasitic life stages, as did the nematodes growing on the wild type and on max4-1 mutant. Surprisingly, the development of females on the $\max 2-1$ and the max4-1 mutant was even enhanced compared to the control based on their body size and the production of a gelatinous egg matrix. This points at favorable nutrient conditions, which is consistent with the observation that these females were attached to feeding sites that were better developed than in the wild type control. It thus seems that SLs also play a role in the regulation of the sedentary stage of cyst nematode parasitism, by modulating the development of the syncytium. A similar multifunctional role of SLs was postulated for the interaction between parasitic plants and their host. SLs induce the germination of parasitic plants, but also seem to play a role in the regulation of the attachment, possibly through an effect on the establishment of a xylem bridge inside the root vasculature for the uptake of nutrients (HeideJørgensen and Kuijt 1995; Jamil et al. 2011). Indeed, a tomato strigolactone mutant that was infected with pregerminated Phelipanche ramosa seeds displayed a lower resistance towards infection showing that strigolactones positively affect resistance (Cheng et al. 2017). This is consistent with the better development of females in the max2-1 and max4-1 mutants of Arabidopsis as found in the present study. Interestingly, sedentary endoparasitic nematodes and plant-parasitic plants share remarkable characteristics in their mode of action during parasitism of plant roots (Mitsumasu et al. 2015), including as it seems now, the effect of SLs in different pre- and parasitic life stages.

Together, our data show that SLs play distinct roles in cyst nematode parasitism. Disruption of SL signalling compromises cyst nematode host attraction and subsequent invasion, but, for those nematodes that successfully invade the roots, it enhances growth and development of the feeding structure. Interestingly, opposite roles for SLs was also reported for lateral root (LR) formation in an auxin-dependent manner. SLs - through their effect on polar auxin efflux transporters of the PIN protein family - reduce auxin import to the roots when auxin levels are low, thereby inhibiting LR formation, but when auxin levels are high SLs downregulate this towards a more optimum level for LR production (Ruyter-Spira et al. 2011). Like LR formation, the induction and expansion of cyst nematode syncytia is mediated by the accumulation of the hormone auxin (Goverse et al. 2000). This accumulation occurs in an early stage of the feeding cell development (Karczmarek et al. 2004) and is the result of nematode-induced manipulation of the PIN protein family (Grunewald et al. 2009). SL induced changes in auxin homeostasis at the nematode feeding site are possibly responsible for the observed enhanced development of the syncytia in the max2-1 and max4-1 mutants. Additional molecular research is required to further study the cross talk between SLs and auxin during cyst nematode feeding cell formation. This will not only provide novel insights in the mechanisms underlying cyst nematode feeding cell formation, but also in the chemical communication between cyst nematodes and their host plants in the rhizosphere.

Acknowledgements We are grateful to Ottoline Leyser who supplied us with seeds of the $\max 2-1$ and $\max 4-1$ Arabidopsis lines and the Erasmus Mundus Master Programme EUMAINE for the support of CMEM.

Compliance with ethical standards There are no conflicts of interest and the manuscript does not involve Human Participants or Animals. All authors are informed and agree on the publication of the manuscript.

OpenAccess This article is distributed under the terms of the Creative Commons Attribution 4.0 International License (http:// creativecommons.org/licenses/by/4.0/), which permits unrestricted use, distribution, and reproduction in any medium, provided you give appropriate credit to the original author(s) and the source, provide a link to the Creative Commons license, and indicate if changes were made. 


\section{References}

Abad, P., Gouzy, J., Aury, J. M., Castagnone-Sereno, P., Danchin, E. G., Deleury, E., et al. (2008). Genome sequence of the metazoan plant-parasitic nematode Meloidogyne incognita. Nature Biotechnology, 26(8), 909-915. https://doi.org/10.1038/nbt.1482.

Akhkha, A., Kusel, J., Kennedy, M., \& Curtis, R. (2002). Effects of phytohormones on the surfaces of plant-parasitic nematodes. Parasitology, 125(2), 165-175.

Akintayo, A., Tylka, G. L., Singh, A. K., Ganapathysubramanian, B., Singh, A., \& Sarkar, S. (2018). A deep learning framework to discern and count microscopic nematode eggs. Scientific Reports, 8(1), 9145. https://doi.org/10.1038 /s41598-018-27272-w.

Akiyama, K., Matsuzaki, K., \& Hayashi, H. (2005). Plant sesquiterpenes induce hyphal branching in arbuscular mycorrhizal fungi. Nature, 435(7043), 824-827. https://doi.org/10.1038 /nature 03608 .

Al-Babili, S., \& Bouwmeester, H. J. (2015). Strigolactones, a novel carotenoid-derived plant hormone. Annual Review of Plant Biology, 66, 161-186. https://doi.org/10.1146/annurevarplant-043014-114759.

Cabrera, J., Diaz-Manzano, F. E., Fenoll, C., \& Escobar, C. (2015). Developmental pathways mediated by hormones in nematode feeding sites. In C. Escobar, \& C. Fenoll (Eds.), Plant nematode interactions : A view on compatible interrelationships (Vol. 73, pp. 167-188, Advances in Botanical Research). London, UK: Academic Press Ltd-Elsevier Ltd.

Cheng, X., Flokova, K., Bouwmeester, H., \& Ruyter-Spira, C. (2017). The role of endogenous strigolactones and their interaction with $\mathrm{ABA}$ during the infection process of the parasitic weed Phelipanche ramosa in tomato plants. Frontiers in Plant Science, 8, 392. https://doi.org/10.3389 /fpls.2017.00392.

Clarke, A. J., \& Shepherd, A. M. (1964). Synthetic hatching agents for Heterodera schachtii Schm. and their mode of action. Nematologica, 10(3), 431-453. https://doi. org/10.1163/187529264x00457.

Cook, C. E., Whichard, L. P., Turner, B., Wall, M. E., \& Egley, G. H. (1966). Germination of Witchweed (Striga lutea Lour.): Isolation and properties of a potent stimulant. Science, 154(3753), 1189-1190. https://doi.org/10.1126/science.154.3753.1189.

Cook, C. E., Whichard, L. P., Wall, M., Egley, G. H., Coggon, P., Luhan, P. A., \& McPhail, A. T. (1972). Germination stimulants. II. Structure of strigol, a potent seed germination stimulant for witchweed (Striga lutea). Journal of the American Chemical Society, 94(17), 6198-6199. https://doi. org/10.1021/ja00772a048.

Curtis, R. H. (2008). Plant-nematode interactions: Environmental signals detected by the nematode's chemosensory organs control changes in the surface cuticle and behaviour. Parasite, 15(3), 310-316. https://doi.org/10.1051 /parasite/2008153310.

de Almeida Engler, J., Vieira, P., Rodiuc, N., de Sa, M. F. G., \& Engler, G. (2015). The Plant Cell Cycle Machinery: Usurped and Modulated by Plant-Parasitic Nematodes. In C. Escobar,
\& C. Fenoll (Eds.), Plant Nematode Interactions: A View on Compatible Interrelationships (Vol. 73, pp. 91-118, Advances in Botanical Research). London, UK: Academic Press Ltd-Elsevier Ltd.

Dube, M. P., \& Olivier, A. (2001). Striga gesnerioides and its host, cowpea: Interactions and control methods. Canadian Journal of Botany, 79, 1225-1240.

Duncan, L. H., Robertson, W. M., Kusel, R. J., \& Phillips, M. S. (1996). A putative nematode auxin binding protein from the potato cyst nematode Globodera pallida. Nematropica, 26, 259.

Golinowski, W., Grundler, F. M. W., \& Sobczak, M. (1996). Changes in the structure of Arabidopsis thaliana during female development of the plant-parasitic nematode Heterodera schachtii. Protoplasma, 194(1-2), 103-116. https://doi.org/10.1007/Bf01273172.

Gomez-Roldan, V., Fermas, S., Brewer, P. B., Puech-Pages, V., Dun, E. A., Pillot, J. P., et al. (2008). Strigolactone inhibition of shoot branching. Nature, 455(7210), 189-194. https://doi.org/10.1038/nature07271.

Goverse, A., \& Bird, D. (2011). The role of plant hormones in nematode feeding cell formation. In J. Jones, G. Gheysen, \& C. Fenoll (Eds.), Genomics and molecular genetics of plantnematode interactions (pp. 325-347). Dordrecht: Springer Netherlands.

Goverse, A., Overmars, H., Engelbertink, J., Schots, A., Bakker, J., \& Helder, J. (2000). Both induction and morphogenesis of cyst nematode feeding cells are mediated by auxin. Molecular Plant-Microbe Interactions, 13(10), 1121-1129. https://oi.org/10.1094/MPMI.2000.13.10.1121.

Grundler, F. M. W., \& Wyss, U. (1992). Seminar: Heterodera schachtii and Arabidopsis thaliana, a model hostparasite interaction. Nematologica, 38(1-4), 488-493. https://doi.org/10.1163/187529292X00450.

Grundler, F., Schnibbe, L., \& Wyss, U. (1991). In vitro studies on the behavior of second-stage juveniles of Heterodera schachtii (Nematoda: Heteroderidae) in response to host plant-root exudates. Parasitology, 103, 149-155. https://doi.org/10.1017/S0031182000059394.

Grunewald, W., Cannoot, B., Friml, J., \& Gheysen, G. (2009). Parasitic nematodes modulate PIN-mediated auxin transport to facilitate infection. PLoS Pathogens, 5(1), e1000266. https://doi.org/10.1371/journal.ppat.1000266.

Heide-Jørgensen, H. S., \& Kuijt, J. (1995). The haustorium of the root parasite Triphysaria (Scrophulariaceae), with special reference to xylem bridge ultrastructure. American Journal of Botany, 82(6), 782-797. https://doi.org/10.1002/j.15372197.1995.tb15691.x.

Hofmann, J., Youssef-Banora, M., de Almeida Engler, J., \& Grundler, F. M. W. (2010). The role of callose deposition along plasmodesmata in nematode feeding sites. Molecular Plant-Microbe Interactions, 23(5), 549557. https://doi.org/10.1094/mpmi-23-5-0549.

Holtmann, B., Kleine, M., \& Grundler, F. M. W. (2000). Ultrastructure and anatomy of nematode-induced syncytia in roots of susceptible and resistant sugar beet. Protoplasma, 211(1-2), 39-50. https://doi.org/10.1007/Bf01279898.

Ito, D., Toyota, K., \& Sedrati, G. M. (2015). Suppression of the soybean cyst nematode, Heterodera glycines, using water 
extract of bean sprout residue. Nematology, 17(5), 523-530. https://doi.org/10.1163/15685411-00002886.

Jamil, M., Charnikhova, T., Cardoso, C., Jamil, T., Ueno, K., Verstappen, F., et al. (2011). Quantification of the relationship between strigolactones and Striga hermonthica infection in rice under varying levels of nitrogen and phosphorus. Weed Research, 51(4), 373-385. https://doi.org/10.1111/j.13653180.2011.00847.x.

Jones, J. T., Haegeman, A., Danchin, E. G., Gaur, H. S., Helder, J., Jones, M. G., et al. (2013). Top 10 plant-parasitic nematodes in molecular plant pathology. Molecular Plant Pathology, 14(9), 946-961. https://doi.org/10.1111/mpp.12057.

Kapulnik, Y., Delaux, P. M., Resnick, N., Mayzlish-Gati, E., Wininger, S., Bhattacharya, C., Séjalon-Delmas, N., Combier, J. P., Bécard, G., Belausov, E., Beeckman, T., Dor, E., Hershenhorn, J., \& Koltai, H. (2011). Strigolactones affect lateral root formation and root-hair elongation in Arabidopsis. Planta, 233(1), 209-216. https://doi. org/10.1007/s00425-010-1310-y.

Karczmarek, A., Overmars, H., Helder, J., \& Goverse, A. (2004). Feeding cell development by cyst and root-knot nematodes involves a similar early, local and transient activation of a specific auxin-inducible promoter element. Molecular Plant Pathology, 5(4), 343-346. https://doi.org/10.1111/j.13643703.2004.00230.x.

Kohlen, W., Charnikhova, T., Liu, Q., Bours, R., Domagalska, M. A., Beguerie, S., Verstappen, F., Leyser, O., Bouwmeester, H., \& Ruyter-Spira, C. (2011a). Strigolactones are transported through the xylem and play a key role in shoot architectural response to phosphate deficiency in nonarbuscular mycorrhizal host Arabidopsis. Plant Physiology, 155(2), 974-987. https://doi.org/10.1104/pp.110.164640.

Kohlen, W., Ruyter-Spira, C., \& Bouwmeester, H. J. (2011b). Strigolactones: A new musician in the orchestra of plant hormones. Botany, 89(12), 827-840. https://doi.org/10.1139 /b11-063.

Koltai, H. (2015). Cellular events of strigolactone signalling and their crosstalk with auxin in roots. Journal of Experimental Botany, 66(16), 4855-4861. https://doi.org/10.1093 /jxb/erv178.

Kyndt, T., Goverse, A., Haegeman, A., Warmerdam, S., Wanjau, C., Jahani, M., Engler, G., de Almeida Engler, J., \& Gheysen, G. (2016). Redirection of auxin flow in Arabidopsis thaliana roots after infection by root-knot nematodes. Journal of Experimental Botany, 67(15), 4559-4570. https://doi.org/10.1093/jxb/erw230.

López-Ráez, J. A., Charnikhova, T., Gomez-Roldan, V., Matusova, R., Kohlen, W., De Vos, R., et al. (2008). Tomato strigolactones are derived from carotenoids and their biosynthesis is promoted by phosphate starvation. The New Phytologist, 178(4), 863-874. https://doi.org/10.1111/j.14698137.2008.02406.x.

Mabrouk, Y., Zourgui, 1., Sifi, B., Delavault, P., Simier, P., \& Belhadj, O. (2007). Some compatible Rhizobium leguminosarum strains in peas decrease infections when parasitised by Orobanche crenata. Weed Research, 47(1), 44-53. https://doi.org/10.1111/j.1365-3180.2007.00548.x.

Masamune, T. (1976). Purification of the hatching substance of the soybean cyst nematode. Abstract of special lecture at $34^{\text {th }}$ annual meeting (p. 91). Chemical Society of Japan.

Masamune, T., Anetai, M., Takasugi, M., \& Katsui, N. (1982). Isolation of a natural hatching stimulus, Glycinoeclepin-a, for the soybean cyst nematode. Nature, 297(5866), 495-496. https://doi.org/10.1038/297495a0.

Mitsumasu, K., Seto, Y., \& Yoshida, S. (2015). Apoplastic interactions between plants and plant root intruders. Frontiers in Plant Science, 6, 617. https://doi.org/10.3389/fpls.2015.00617.

Peláez-Vico, M. A., Bernabeu-Roda, L., Kohlen, W., Soto, M. J., \& López-Ráez, J. A. (2016). Strigolactones in the rhizobiumlegume symbiosis: Stimulatory effect on bacterial surface motility and down-regulation of their levels in nodulated plants. Plant Science, 245, 119-127. https://doi.org/10.1016 /j.plantsci.2016.01.012.

Postma, W. J., Slootweg, E. J., Rehman, S., Finkers-Tomczak, A., Tytgat, T. O., van Gelderen, K., et al. (2012). The effector SPRYSEC-19 of Globodera rostochiensis suppresses CC-NBLRR-mediated disease resistance in plants. Plant Physiology, 160(2), 944-954. https://doi.org/10.1104/pp.112.200188.

Riga, E., Topp, E., Potter, J., Welacky, T., Anderson, T., \& Tenuta, A. (2001). The impact of plant residues on the soybean cyst nematode, Heterodera glycines. Canadian Journal of Plant Pathology, 23(2), 168-173. https://doi.org/10.1080 /07060660109506926.

Robinson, F., \& Perry, R. (2006). Behaviour and sensory perception. In R. N. Perry \& M. Moens (Eds.), Plant nematology (pp. 211-233). Wallingford, UK: CABI Publishing.

Rodiuc, N., Vieira, P., Banora, M. Y., \& de Almeida Engler, J. (2014). On the track of transfer cell formation by specialized plant-parasitic nematodes. Frontiers in Plant Science, 5, 160. https://doi.org/10.3389/fpls.2014.00160.

Ruyter-Spira, C., Kohlen, W., Charnikhova, T., van Zeijl, A., van Bezouwen, L., de Ruijter, N., Cardoso, C., Lopez-Raez, J. A., Matusova, R., Bours, R., Verstappen, F., \& Bouwmeester, H. (2011). Physiological effects of the synthetic strigolactone analog GR24 on root system architecture in Arabidopsis: Another belowground role for strigolactones? Plant Physiology, 155(2), 721-734. https://doi.org/10.1104 /pp.110.166645.

Sijmons, P. C., Grundler, F. M. W., von Mende, N., Burrows, P. R., \& Wyss, U. (1991). Arabidopsis thaliana as a new model host for plant-parasitic nematodes. The Plant Journal, 1(2), 245254. https://doi.org/10.1111/j.1365-313X.1991.00245.x.

Sobczak, M., \& Golinowski, W. (2011). Cyst nematodes and syncytia. In J. Jones, G. Gheysen, \& C. Fenoll (Eds.), Genomics and molecular genetics of plant-nematode interactions (pp. 61-82). Dordrecht: Springer Netherlands.

Sorefan, K., Booker, J., Haurogne, K., Goussot, M., Bainbridge, K., Foo, E., et al. (2003). MAX4 and RMS1 are orthologous dioxygenase-like genes that regulate shoot branching in Arabidopsis and pea. Genes \& Development, 17(12), 14691474. https://doi.org/10.1101/gad.256603.

Steele, A. E. (1972). Evaluation of cyst selection as a means of reducing variation in sugar beet nematode inocula. Journal American Society Sugar Beet Technology, 17, 22-29.

Stirnberg, P., van De Sande, K., \& Leyser, H. M. (2002). MAX1 and MAX2 control shoot lateral branching in Arabidopsis. Development, 129(5), 1131-1141. 
Turner, S. J., \& Rowe, A. R. (2006). Cyst nematodes. In R. N. Perry \& M. Moens (Eds.), Plant nematology (pp. 91-122). Wallingford, UK: CABI Publishing.

Tytgat, T., De Meutter, J., Vanholme, B., Claeys, M., Verreijdt, L., Gheysen, G., et al. (2002). Development and pharyngeal gland activities of Heterodera schachtii infecting Arabidopsis thaliana roots. Nematology, 4, 899-908. https://doi.org/10.1163/156854102321122511.

Umehara, M., Hanada, A., Yoshida, S., Akiyama, K., Arite, T., Takeda-Kamiya, N., Magome, H., Kamiya, Y., Shirasu, K., Yoneyama, K., Kyozuka, J., \& Yamaguchi, S. (2008). Inhibition of shoot branching by new terpenoid plant hormones. Nature, 455(7210), 195-200. https://doi. org/10.1038/nature07272.

von Mende, N. (1997). Invasion and migration behaviour of sedentary nematodes. In C. Fenoll, F. M. W. Grundler, \& S. A. Ohl (Eds.), Cellular and molecular aspects of plantnematode interactions (pp. 51-64). Dordrecht: Springer Netherlands.

Westerdijk, J. (1917). De nieuwe wegen van het phytopathologisch onderzoek. Inaugurele rede. Universiteit Utrecht.

Wigchert, S. C., \& Zwanenburg, B. (1999). A critical account on the inception of Striga seed germination. Journal of Agricultural and Food Chemistry, 47(4), 1320-1325. 